\title{
Normalized Spray Flux Distribution in Impinging Atomization*
}

\author{
Chihiro Inoue, ${ }^{1) \dagger}$ Junya Kouwa, ${ }^{2)}$ Toshinori Watanabe, ${ }^{1)}$ Takehiro Himeno, ${ }^{1)}$ \\ Seiji UZAWA, ${ }^{1)}$ and Shinsuke MATSUNO ${ }^{2)}$ \\ ${ }^{1)}$ Department of Aeronautics and Astronautics, The University of Tokyo, Tokyo 113-8656, Japan \\ ${ }^{2}$ IHI Corporation, Kanagawa 235-8501, Japan
}

Key Words: Impinging Injector, Atomization, Spray Flux Distribution, Patternator, Flow Visualization

\author{
Nomenclature \\ $d$ : droplet diameter [m] \\ $D$ : nozzle diameter $[\mathrm{m}]$ \\ $g$ : gravitational acceleration $\left[\mathrm{m} / \mathrm{s}^{2}\right]$ \\ $L$ : length of a continuous sheet $[\mathrm{m}]$ \\ $\dot{m}$ : injection mass flow rate $[\mathrm{kg} / \mathrm{s}]$ \\ $\dot{q}$ : spray mass flux $\left[\mathrm{kg} / \mathrm{s} / \mathrm{m}^{2}\right]$ \\ $\overline{\dot{q}}$ : normalized spray mass flux \\ $v$ : droplet velocity $[\mathrm{m} / \mathrm{s}]$ \\ $V$ : liquid injection velocity $[\mathrm{m} / \mathrm{s}]$ \\ $W e$ : Weber number $\left(\rho V^{2} D / \sigma\right)$ \\ $\delta$ : distance where atomization is completed from the sheet \\ edge $[\mathrm{m}]$ \\ $\mu$ : coefficient of viscosity $[\mathrm{Pa} \cdot \mathrm{s}]$ \\ $\rho$ : density $\left[\mathrm{kg} / \mathrm{m}^{3}\right]$ \\ $\sigma$ : surface tension coefficient $[\mathrm{N} / \mathrm{m}]$
}

\section{Introduction}

It is well known that both thrust performance and combustion stability are highly dependent on the atomization and mixing of propellants in the combustion chamber of a liquid propulsion system. ${ }^{1-3)}$ Several different propellant injection systems have been proposed for use with various combinations of liquid propellants and put to practical applications. ${ }^{1,4)}$ Impinging jet injectors are the preferred type, especially in the case of bi-propellant thrusters. Through the impingement of two jets against one another, a sheet of liquid is generated that in turn produces both ligaments and droplets. When evaluating such injectors, it is important to obtain accurate information regarding the droplet size, spray flux distribution and local mixing ratios. A conventional technique for measuring the spray flux distribution involves the use of mechanical or optical patternators. A mechanical patternator directly catches the sprays using a bundle of collection vessels. ${ }^{5,6)}$ This is a reliable method, although it is time consuming and has difficulty acquiring highly resolved spatial data. An optical patternator employing a phase Doppler interferometer is useful for the non-contact measurement of

(C) 2017 The Japan Society for Aeronautical and Space Sciences

*Received 22 May 2016; final revision received 25 November 2016; accepted for publication 14 February 2017.

†Corresponding author, inoue@ rocketlab.t.u-tokyo.ac.jp sprays containing only spherical droplets. ${ }^{7-9)}$ In this research, we investigate a new framework for estimating spray flux distributions from a limited number of measurement points in the case of impingement atomization, and examine its applicability. From visualization results, we are able to determine the time-integrated pathways of droplets and the spread of the spray. Using the spray flux distribution across a given plane, together with the spread of the spray, a normalized spray flux can be defined based on the injection conditions and the distance from the impingement point. From the normalized distribution, we can calculate the dimensional distribution at any other location.

\section{Experimental Method}

Figure 1 presents a diagram of the experimental apparatus. Two reservoirs are used to contain the working fluid (water) under pressure. This liquid is injected into still air from two nozzles, as shown in more detail in Fig. 2. These nozzles are precision-made glass tubes with a diameter, $D$, of $1 \mathrm{~mm}$ and are positioned to give an apex angle $(2 \theta)$ of $40^{\circ}$. The two liquid jets impinge to create a liquid sheet. The origin of the coordinate system $(x, y, z)$ is located at the impingement point and the $x$-axis and $y$-axis are assigned to the perpendicular and transverse directions relative to the liquid sheet, respectively. The nozzles are adjustable to eliminate any misalignment of the water jets. Table 1 summarizes the physical properties of the working fluids in this system (water and air). The surface tension coefficient was measured using an automatic surface tensiometer employing the plate method (CBVP-Z, Kyowa Interface Science Co., Ltd.). The atomization process was visualized using a high-speed video camera (Photron FASTCAM APX-RS) in conjunction with a backlighting technique. Typically, the frame rate and shutter speed were set at 10,000 fps and 1/100,000 s, respectively. The 2D spray mass flux distribution was determined using a measurement system based on a mechanical patternator (Fig. 3). Figure 4 shows the patternator, which was manufactured using a 3D printer. The spray mass flux distribution in the $x-y$ plane was measured at several locations along the $z$ axis. In this technique, the various droplets passed through specific sections of the patternator and were collected in the corresponding test tubes (see Fig. 3). The spray mass flux was then calculated based on the net mass of liquid in each 


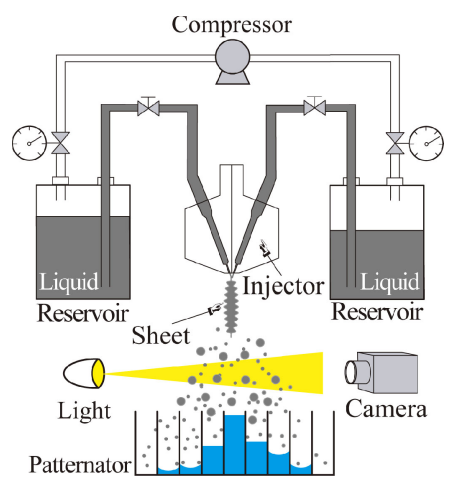

Fig. 1. Overview of the experimental apparatus.

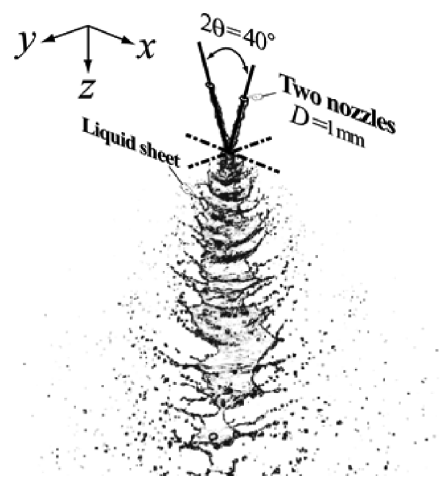

Fig. 2. Detailed view near the impingement point.

Table 1. Physical properties of the working fluids.

\begin{tabular}{lccc}
\hline Fluid & $\rho\left[\mathrm{kg} / \mathrm{m}^{3}\right]$ & $\mu[\mathrm{Pa} \cdot \mathrm{s}]$ & $\sigma[\mathrm{mN} / \mathrm{m}]$ \\
\cline { 1 - 3 } Air & 1.2 & $1.8 \times 10^{-5}$ & \multirow{2}{*}{$72.4 \pm 0.4$} \\
\cline { 1 - 3 } Water & 1000 & $1.0 \times 10^{-3}$ & \\
\hline
\end{tabular}

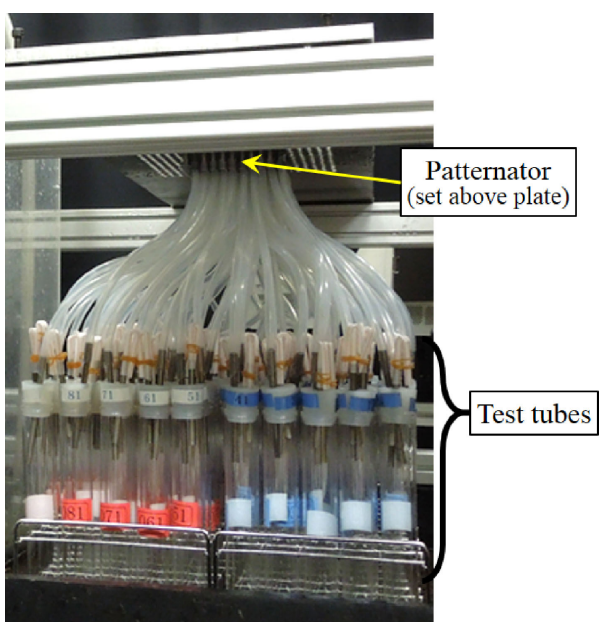

Fig. 3. Spray distribution measurement system set beneath the injector.

test tube divided by the trial time span and the area of the measurement section. The patternator covered an area in the $x-y$ plane of $100 \times 100 \mathrm{~mm}$ with a spatial resolution of $10 \times 10 \mathrm{~mm}$. The patternator contained a total of $100 \mathrm{sec}-$ tions. Through experimental trials, it was confirmed that the relative error in the spray mass flux was less than $1 \%$.

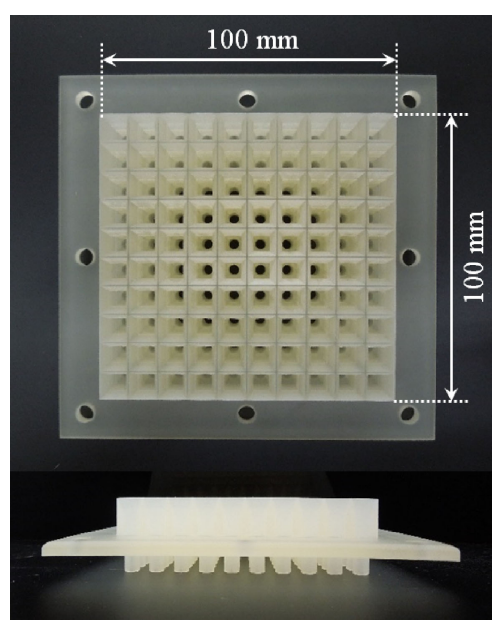

Fig. 4. The 3D-printed patternator.

Top and side view. Each section is connected to the test tubes downstream.

All experiments were conducted at $W e=3,600$, with $D=1 \mathrm{~mm}$ and $V=16 \mathrm{~m} / \mathrm{s}$, corresponding to a practical situation for which $W e \geq 10^{3}$.

\section{Results and Discussions}

Figure 5 shows the results obtained from flow visualization tests, in which an instantaneous image is placed over a time-integrated image acquired for a duration of $10 \mathrm{~ms}$. Each black dashed line indicates the path of an individual droplet. The impingement of the two liquid jets is seen to generate an undulating liquid sheet that extends from the stagnation point, with ligaments elongating to the point of fragmentation at the sides and bottom of the sheet in the $y-z$ plane. The $L / D$ value of the sheet in the $z$ direction is approximately 40 , which is consistent with past experimental results. ${ }^{10)}$ The droplets spread out in straight lines to the left and right in an oblique downward direction. In addition, in the $x-z$ plane, the droplets fly straight out from the sheet. The outer edge of the spray consists of droplets fragmented close to the impingement point. We can also observe that the droplets spread out, starting from the impingement point, despite the presence of the continuous sheet.

An overview of the spray mass flux distributions is presented in Fig. 6, as determined at several locations along the $z$ axis. Here, regions having greater than $1 \%$ of the total flow rate through each plane are illustrated. A dense spray is obviously present beneath the impingement point and the spray area then widens, moving downstream. The spray naturally spreads out in the $y$ direction rather than in the $x$ direction. In the planes beyond $z=125 \mathrm{~mm}$, the spray extends beyond the area of the patternator in the $y$ direction, and so the edge of the spray is not measured. Figure 7 shows the spray mass flux distribution along the $y$ axis at $x=0 \mathrm{~mm}$. At each distance from the impingement point, the mass flux is highest at $y=0 \mathrm{~mm}$ and is smaller around the edges. Although these distributions include both ligaments and droplets, the atomization process is complete and all droplets 

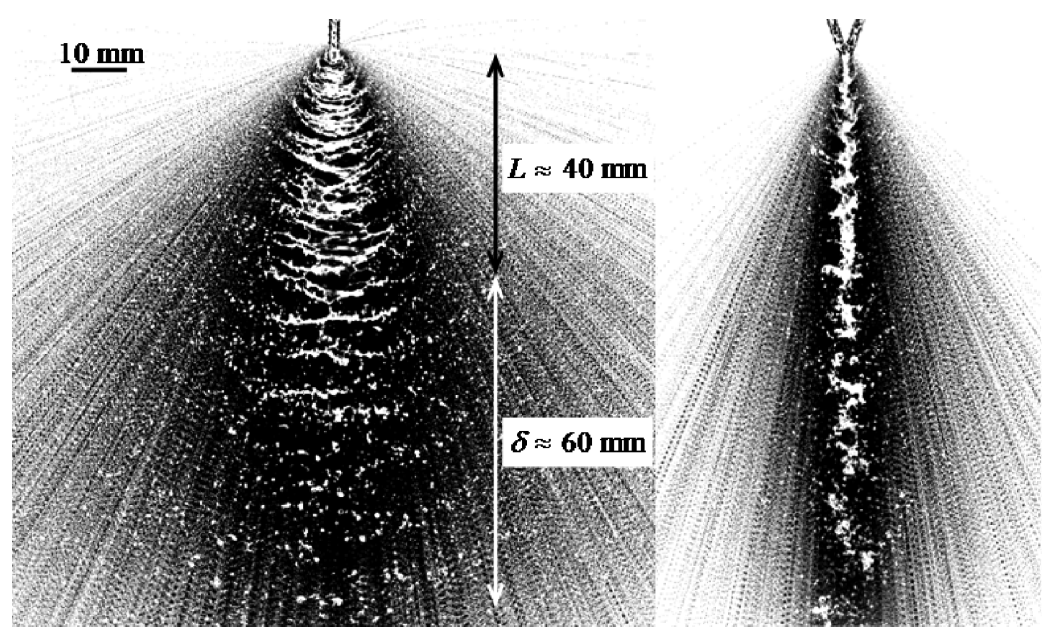

Fig. 5. Time-integrated image (black) overlapped with an instantaneous image (white). Left: Front view in the $y-z$ plane, Right: Side view in the $x-z$ plane.

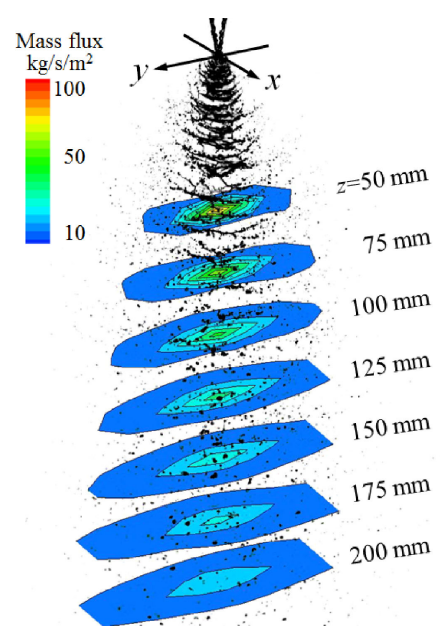

Fig. 6. Distribution of the spray mass flux at $z=50-200 \mathrm{~mm}$ below the impingement point. become spherical at $z>L+\delta$. In impinging atomization, the diameter and velocity of the large droplets are roughly equal to $D$ and $V$, respectively (see Appendix). Thus, the capillary time scale $\tau_{\sigma} \sim \sqrt{\rho D^{3} / \sigma}$ gives the time delay for a droplet to fragment from a ligament, corresponding to $\tau_{\sigma}=\delta / V$, and so $\delta$ can be simply expressed as $\delta / D \sim \sqrt{W e}$. All droplets are spherical and will spread out in straight lines in the region defined by

$$
\frac{z}{D}>\frac{L}{D}+\sqrt{W e}
$$

By substituting $L / D \approx 40$ and $W e=3,600$ corresponding $\delta / D \approx 60, z / D>100$ is obtained.

If we assume that the droplets spread out from the impingement point in the $x-y$ plane roughly in the same manner as along the $z$ axis (Fig. 5), the cross-sectional area of the droplet "streamtube," consisting of droplet path lines, widens in proportion to $z^{2}$. In this case, a normalized flux can be newly defined against the normalized $y / z$ (or $x / z)$ location as

$$
\overline{\dot{q}}=\frac{\dot{q} z^{2}}{\dot{m}} \text {. }
$$

The effects of droplet evaporation and gravity are ignored in this case, because the liquid is at room temperature and because $z \ll V^{2} / g$ at approximately $10 \mathrm{~m}$. It should be noted that the relationship in Eq. (2) is not universal, but rather depends on the spreading pattern of the spray. Figure 8 shows the experimental results by plotting $\overline{\dot{q}}$ against $y / z$, and confirms that the spray flux at $z / D>100$ is consistently normalized. In the region defined by $z / D<100$, however, ligaments and highly deformed large droplets remain, and the flux distribution has not yet converged. By fitting the data to a converged distribution, we obtain an equation, for example,

$$
\overline{\dot{q}}=25 \exp \left(-(y / z)^{2} / 0.03\right)
$$

Since we know $\dot{m}=0.025 \mathrm{~kg} / \mathrm{m}^{3}$, we can determine $\dot{q}$ from Eqs. (2) and (3). In Fig. 9, the experimental $\dot{q}$ values and the corresponding calculation results at $z=100,150$ and

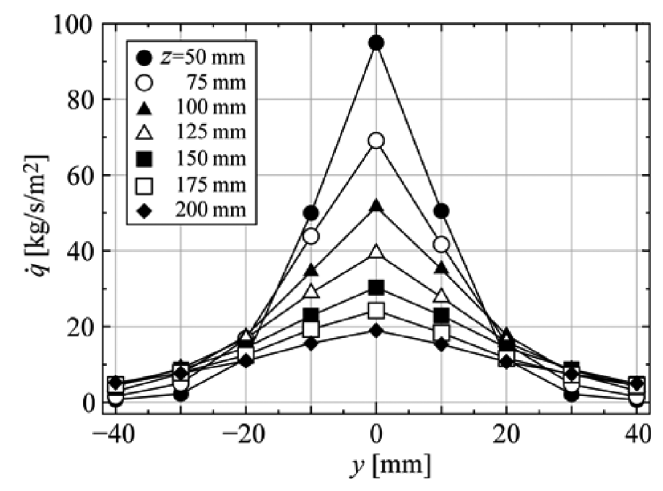

Fig. 7. Distribution of the spray mass flux in the $y$ direction at $x=0 \mathrm{~mm}$. The symbols indicate the centers of sections in the patternator. Measurement area is $-50 \mathrm{~mm}<y<50 \mathrm{~mm}$.

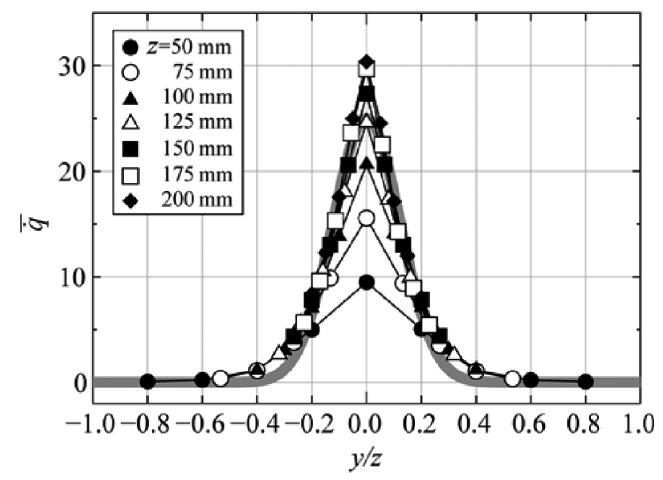

Fig. 8. Normalized spray mass flux distributions. The symbols are experimental results and the gray bold line is obtained from Eq. (3).

$200 \mathrm{~mm}$ are presented. These plots confirm the reproducibility of the data at $z=150$ and $200 \mathrm{~mm}$ with a maximum error of $10 \%$ at $y=0 \mathrm{~mm}$. At $z=100 \mathrm{~mm}$, we see that the value calculated is $20 \%$ greater than the experimental result at $y=0 \mathrm{~mm}$. At this point, the spray spreads out according to $z^{n}$, where $n<2$ rather than $z^{2}$ due to the shrinkage of ligaments in the $y$ direction beneath the sheet. These results verify the applicability of the present framework, provided that 


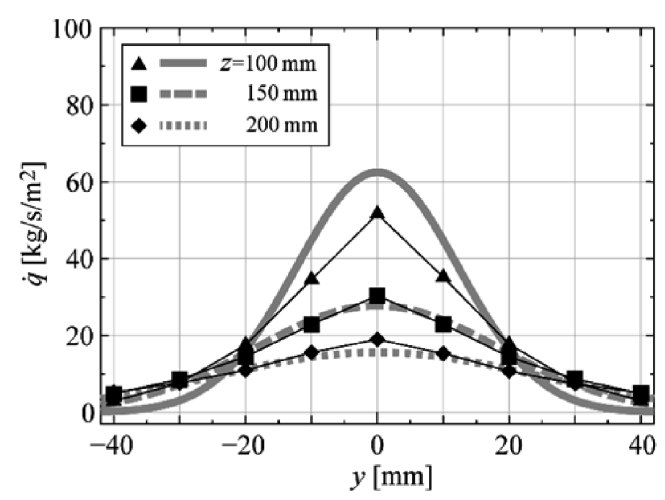

Fig. 9. Comparison of calculated results (gray lines) with experimental data (symbols) at $x=0 \mathrm{~mm}$.

The experimental results are the same as those in Fig. 7.

the spray is within the range over which the normalization is valid. It is expected that this technique will also be useful as a means of estimating detailed upstream 2D distributions on the basis of easily measured coarse downstream distributions.

\section{Conclusions}

A simple method of determining normalizing spray flux distribution was demonstrated applying flow visualization and the measurement of droplet distribution using a patternator. The conclusions can be summarized as follows.

(1) The 3D-printed patternator was demonstrated to be useful for the 2D measurement of spray mass flux distributions.

(2) The spray mass flux distribution was normalized as a function of normalized distance after atomization was complete.

(3) The flux distribution at any location could be calculated from the normalized distribution convergence considering the spread of the spray.

Future work is required to validate the general applicability of this framework.

\section{Acknowledgments}

This work was partially supported by JSPS KAKENHI Grant Number 15K14246.

\section{References}

1) Sutton, G. P. and Biblarz, O.: Rocket Propulsion Elements, 8th ed., John Wiley \& Sons, Inc., New Jersey, 2010.

2) Lefebvre, A. H.: Atomization and Sprays, Taylor and Francis, New York, 1989.

3) Mayer, W. and Tamura, H.: Propellant Injection in a Liquid Oxygen/ Gaseous Hydrogen Rocket Engine, J. Propul. Power, 12, 6 (1996), pp. 1137-1147.

4) Gill, G. S.: Liquid Rocket Engine Injectors, NASA SP-8089, 1976.

5) McVey, J. B., Russell, S., and Kennedy, J. B.: High-Resolusion Patternator for the Characterization of Fuel Sprays, J. Propul. Power, 3, 3 (1987), pp. 202-209.

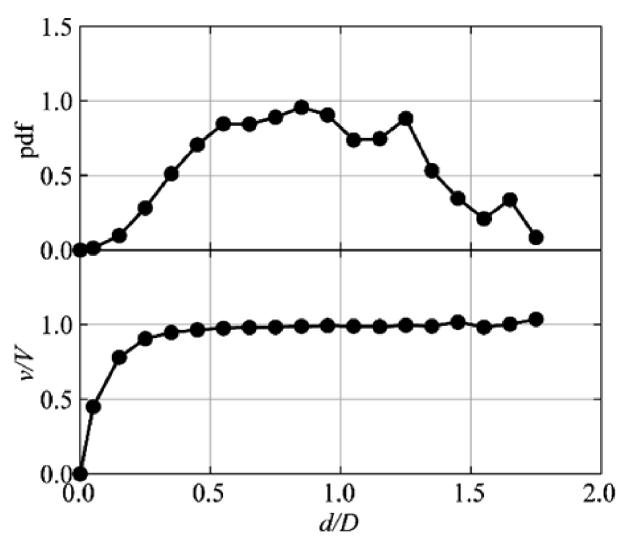

Fig. 10. The pdf of droplet volumes and velocities. The results are plotted at every $d / D=0.1$. The data is $99 \%$ of the cumulative number of all droplets.

6) Ashgriz, N., Brocklehurst, W., and Tally, D.: Mixing Mechanisms in a Pair of Impinging Jets, J. Propul. Power, 17, 3 (2001), pp. 736-749.

7) Feikema, D. A.: Optical Measurements in Rocket Engine Liquid Sprays, NASA/ASEE Summer Faculty Fellowship Program, 1994, pp. $1-5$.

8) Jung, K., Lim, B., and Yoon, Y.: Comparison of Mixing Characteristics of Unlike Triplet Injectors Using Optical Patternator, J. Propul. Power, 21, 3 (2005), pp. 442-449.

9) Yuan, T., Chen, C., and Huang, B.: Optical Observation of the Impingements of Nitrogen Tetroxide/Monomethylhydrazine Simulants, AIAA J., 44, 10 (2006), pp. 2259-2266.

10) Anderson, W. E., Ryan, H. M., and Santoro, R. J.: Impinging Jet Atomization, Liquid Rocket Engine Combustion Instability, Chapter 8, Progress in Astronautics and Aeronautics, AIAA, Washington, 1995, pp. 215-246.

\section{Appendix}

Both droplet size and velocity were measured. A doublepulse YAG laser (DANTEC DYNAMICS DualPower 13515) was used as the background light source. A long-distance microscope lens (INFINITY Model K2/SC) was employed to enlarge an $y-z$ region of $4 \times 4 \mathrm{~mm}$ at $z=200 \mathrm{~mm}$, at which point the droplets became spherical. Shadow images of droplets in this region were acquired using a CCD camera (DANTEC DYNAMICS FlowSense 4M MkII). The droplet diameter was obtained as the area-equivalent diameter, while velocity was calculated from the distance the center of gravity moved between two laser pulses. Figure 10 shows the experimentally determined probability density function (pdf) for the volume of droplets proportional to $d^{3}$ and the absolute droplet velocities in the $y-z$ plane. A sufficient number of droplets was measured. These data confirm that large droplets were obtained, for which $d$ was approximately equal to $D$, with velocity values approximately equal to $V$. Since the spray flux is proportional to $d^{3}$, the contribution of smaller droplets with $v$ about $0.5 \mathrm{~V}$ is not significant.

J. R. Hulka

Associate Editor 